\title{
Coherent excitation, incoherent excitation, and adiabatic states*
}

\author{
D. Grischkowsky \\ IBM Thomas J. Watson Research Center, Yorktown Heights, New York 10598
}

(Received 1 December 1975; revised manuscript received 7 April 1976)

\begin{abstract}
Coherent excitation of an atomic excited state occurs during the propagation of near-resonant light pulses and is responsible for the induced polarization. Simultaneously, incoherent excitation occurs due to the relaxation processes described by the absorption coefficient. Here, the theory for the coherent and incoherent excitation is initially presented in terms of the traditional vector model. While a complete understanding of the two-level system is provided by the vector model, it is shown to be incomplete when the problem of directly monitoring the coherent and incoherent excitation is considered. This is because this latter problem involves more than two levels. For this more complicated multilevel problem, adiabatic states are introduced to gain further understanding. The adiabatic states are the stationary states of the atom in the presence of the near-resonant laser field; they help to explain the intimate connection between the coherent excitation and the two-photon resonance. Experimental measurements of the coherent and incoherent excitation associated with near-resonant pulse propagation in $\mathrm{Rb}$ vapor are presented. The double-resonance technique used a relatively strong pulsed dye laser tuned near the $5 S_{1 / 2} \rightarrow 5 P_{1 / 2}$ transition (7948 $\AA$ ) of $\mathrm{Rb}$ to produce the coherent and incoherent excitation, and a weak, tunable cw dye laser tuned in the region of the $5 P_{1 / 2} \rightarrow 6 D_{3 / 2}$ transition (6206 $\AA$ ) to monitor this excitation. In agreement with theory, the experimental results demonstrate that coherent excitation is responsible for two-photon absorption, while the incoherent excitation corresponds to one-photon absorption to the $5 P_{1 / 2}$ state.
\end{abstract}

\section{INTRODUCTION}

Coherent excitation ( $\mathrm{CE}$ ) of atomic transitions is a general phenomenon occuring whenever electromagnetic radiation interacts with atoms. The term $\mathrm{CE}$ designates that excitation of an atomic system for which the induced polarization maintains a definite phase relationship to the driving field. For nonresonant situations CE is usually of relatively minor physical significance; the propagation of light can be adequately described by an appropriate index of refraction and absorption coefficient. However, as the frequency of the light approaches the frequency of an atomic resonance, CE becomes a dominant feature of the interaction between the light and the atomic system. For example, in coherent optics, where the atoms are treated as twolevel systems with excited state $|n\rangle$ and ground state $|2\rangle$, the processes of photon echoes, ${ }^{1}$ self-induced transparency, ${ }^{2}$ optical nutation, ${ }^{3}$ free-induction decay, ${ }^{4}$ adiabatic following, ${ }^{5}$ and adiabatic rapid passage ${ }^{6}$ all involve $C E$ of the atomic system. For these processes large and reversible transfers of energy occur between the electromagnetic wave and the atomic excitation because of $\mathrm{CE}$ and are described by the excited-state population density $N_{n}^{*}(t) .^{7}$ Even in the regime of linear dispersion theory, ${ }^{8-13} \mathrm{CE}$ is very important for nearresonant propagation. Here the group velocity can be obtained from energy-transfer arguments involving the energy density $U_{c}$ of the coherent excitation and the energy density $U_{\mathrm{em}}$ of the electromagnetic wave. Group velocities as slow as $\frac{1}{15} c$ have been observed. ${ }^{13}$ For such a case $U_{c}$ is much larger than $U_{\mathrm{em}}$, and $\mathrm{CE}$ dominates the pulse propagation.

Simultaneous with the coherent excitation, relaxation processes described by the ordinary absorption coefficient cause incoherent excitation of state $|n\rangle$. This excited-state population density $N_{n}(t)$ behaves much differently from CE in that it does not represent energy which can be exchanged back and forth between the atomic system and the propagating electromagnetic wave. Consequently, incoherent excitation has little influence on pulse propagation, other than attenuation. Usually, for the nonresonant problem $N_{n}(t)$ is much larger than $N_{n}^{*}(t)$ due to CE. However, for the near-resonant situation discussed in this paper $N_{n}^{*}(t)$ is more than 10 times larger than $N_{n}(t)$.

The goal of this paper is to gain a precise physical understanding of the coherent and incoherent excitation and for the first time to test this understanding by direct experimental measurements of $N_{n}^{*}(t)$ and $N_{n}(t)$.

The traditional vector model of coherent optics ${ }^{1-6}$ is used in Sec. II to study both coherent and incoherent excitation and to calculate the magnitudes of $N_{n}^{*}(t)$ and $N_{n}(t)$. The vector model, which provides a complete understanding of the two-level system, is seen to be incomplete when one considers the direct measurement of $N_{n}^{*}(t)$ and $N_{n}(t)$ by the absorption of weak probing light to a higher excited state, as illustrated in Fig. 1. The vector model cannot predict the appropriate frequency $\omega_{1}$ for the probing light to monitor either $N_{n}^{*}(t)$ or 


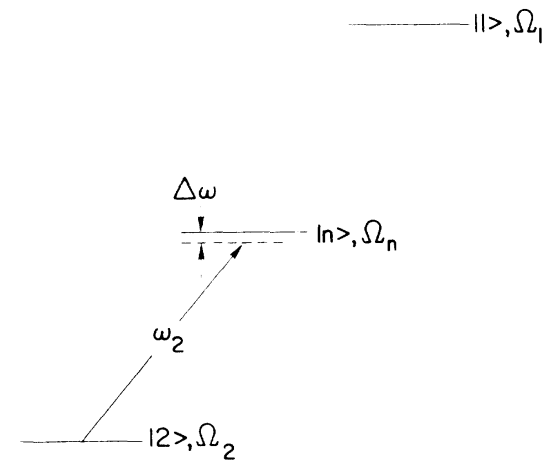

FIG. 1. Energy-level diagram for the double-resonance experiment. A relatively strong dye-laser pulse with frequency $\omega_{2}$ is applied to the system and produces both coherent and incoherent excitation of state $|n\rangle$. The coherent excitation is monitored by the absorption of a weak cw dye laser with frequency $\omega_{1}$ tuned to the twophoton resonance $\omega_{1}+\omega_{2}=\Omega_{12}=\Omega_{1}-\Omega_{2}$, while the incoherent excitation is monitored when $\omega_{1}$ is tuned to the single-photon resonance $\omega_{1}=\Omega_{1 n}$.

$N_{n}(t)$.

The above shortcoming of the vector model, when applied to the multilevel atom, ${ }^{14}$ is overcome by introducing the adiabatic states in Sec. III. The physical idea involved is that the presence of the strong $\overrightarrow{\mathrm{E}}_{2}$ laser pulse changes the atomic Hamiltonian; the adiabatic states are the stationary state $\mathrm{s}^{22-24}$ of this new Hamiltonian. The adiabaticstates analysis confirms that the weak $\overrightarrow{\mathrm{E}}_{1}$ probing beam causes transitions only between the adiabatic state $^{22-28}$ and clarifies the relationship between $\mathrm{CE}$ and the two-photon resonance.

It is shown in Sec. IV and illustrated in Fig. 3 that the coherent excitation $N_{n}^{*}(t)$, calculated by the vector model, is monitored when the frequency $\omega_{1}$ of the probing beam is tuned to the two-photon resonance $\omega_{1}+\omega_{2}=\Omega_{1}-\Omega_{2}=\Omega_{12}$. Thus $N_{n}^{*}(t)$ behaves as an excitation of state $|n\rangle$ but with state $|n\rangle$ having the shifted energy $\omega_{2}+\Omega_{2}$ determined by the driving frequency $\omega_{2}$ and the energy $\Omega_{2}$ of the ground state $|2\rangle$. The incoherent excitation $N_{n}(t)$ is shown to correspond to ordinary excitation of state $|n\rangle$ with energy $\Omega_{n}$ Consequently, $N_{n}(t)$ is monitored when $\omega_{1}$ is tuned to the single-photon resonance $\omega_{1}=\Omega_{1 n}$.

The theory is confirmed by a double-resonance experiment in $\mathrm{Rb}$ vapor, described in Sec. $\mathrm{V}$. Here, a relatively strong pulsed dye-laser beam with frequency $\omega_{2}$ is tuned near the $5 S_{1 / 2}-5 P_{1 / 2}$ transition (7948 $\AA$ ) in $\mathrm{Rb}$ and causes both coherent and incoherent excitation, which are monitored by a weak, tunable cw dye laser with frequency $\omega_{1}$ tuned in the region of the $5 P_{1 / 2}-6 D_{3 / 2}$ transition $(6206 \mathrm{~A})$. In agreement with theory, only the coherent excitation $N_{n}^{*}(t)$ is observed when the fre- quency of the cw laser is tuned to the two-photon resonance $\omega_{1}+\omega_{2}=\Omega_{12}$ corresponding to the $5 S_{1 / 2}$ $-6 D_{3 / 2}$ transition. However, when the $\mathrm{cw}$ laser is tuned to the single-photon resonance corresponding to the $5 P_{1 / 2}-6 D_{3 / 2}$ transition, only the incoherent excitation $N_{n}(t)$ is observed, although $N_{n}^{*}(t)$ is more than 10 times larger than $N_{n}(t)$. This complete experimental separation of $N_{n}^{*}(t)$ and $N_{n}(t)$ occurs because the frequency offset of the pulsed laser with respect to the $5 S_{1 / 2} \rightarrow 5 P_{1 / 2}$ transition is large compared with the inhomogeneous linewidth of this transition. The calculated magnitudes of $N_{n}^{*}(t)$ and $N_{n}(t)$ agree well with the measured values.

\section{TRADITIONAL VECTOR-MODEL THEORY}

For coherent optics ${ }^{1-6}$ the vector model of Feynman, Vernon, and Hellwarth ${ }^{29}$ has been very important for understanding the interaction between atomic systems and resonant or near-resonant light. The model can be applied whenever the frequency of the light is so close to a resonance transition that the atom can be treated as a two-level system. This is the situation for the interaction of the $\overrightarrow{\mathrm{E}}_{2}$ pulsed beam with the atomic system, shown in Fig. 1. Thus it is possible to use the vector model to calculate both the coherent and incoherent excitation following the well-established procedures of coherent optics.

Figure 2(a) illustrates the general vector-model solution for which the pseudomoment $\vec{p}$ precesses about the effective field $\overrightarrow{\mathscr{F}}_{e} \cdot{ }^{1-6,29}$ The figure is drawn in the coordinate frame rotating with angular frequency $\omega_{2}$ about the propagation direction of the light and in which the electric field $\mathscr{S}_{2}$ of the circularly polarized $\overrightarrow{\mathrm{E}}_{2}$ pulse appears stationary. The magnitude of the pseudomoment $\overrightarrow{\mathrm{p}}$ is $|\overrightarrow{\mathrm{p}}|=p_{n 2} / \sqrt{2}$, where $p_{n 2}$ is the magnitude of the electric dipole moment for the $\sigma$ transition between states $|n\rangle$ and $|2\rangle$; the gyroelectric ratio is indicated by $\kappa$, with $\kappa=\sqrt{2} p_{n 2} / \hbar$; the frequency off set $\Delta \omega$ is given by $\Delta \omega=\Omega_{n 2}-\omega_{2}$, where $\Omega_{n 2}=\Omega_{n}-\Omega_{2}$. This notation is connected with earlier work as follows: $N \overrightarrow{\mathrm{p}}$ is the pseudo-electric-dipole-moment of Abella, Kurnit,
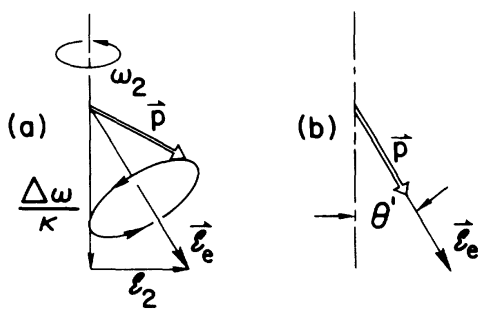

FIG. 2. (a) General solution for the vector model showing the precession of the pseudomoment $\vec{p}$ about the effective field $\overrightarrow{\mathcal{E}}_{e}$. (b) Adiabatic following approximation. 
and Hartmann, ${ }^{1}$ where $N$ is the atomic number density, and $\overrightarrow{\mathrm{p}}$ is $p_{n 2} \sqrt{2} \overrightarrow{\mathrm{r}}$, where $\overrightarrow{\mathrm{r}}$ is the 3 -vector of Feynman, Vernon, and Hellwarth. ${ }^{29}$

The coherent excitation $N_{n}^{*}(t)$ is immediately obtained in terms of the angle $\theta^{\prime}$ between the pseudomoment and the negative precession axis as

$$
N_{n}^{*}(t)=\frac{1}{2} N\left(1-\cos \theta^{\prime}\right),
$$

for any motion of the pseudomoment.

In order to simplify the theoretical and experimental situation, the experiments were conducted so that the adiabatic following approximation was valid. ${ }^{5,13}$ This approximation, illustrated in Fig. 2(b), has the pseudomoment aligned along the instantaneous effective field. For this case and when $\theta^{\prime} \ll 1$, Eq. (1) is well approximated by

$$
N_{n}^{*}(t)=N p_{n 2}^{2} \mathfrak{g}_{2}^{2} / 2 \hbar^{2} \Delta \omega^{2},
$$

which shows the important result that the coherent excitation is proportional to the pulse intensity.

Simultaneous with $\mathrm{CE}$, relaxation processes cause incoherent excitation described by the ordinary absorption coefficient $\alpha_{0}$, which for off-resonant light is given by the vector model $\mathrm{as}^{5,13}$

$$
\alpha_{0}=4 \pi \omega_{2} N p_{n 2}^{2} / c T_{2} \hbar(\Delta \omega)^{2} .
$$

For the experiments discussed here the homogeneous relaxation time $T_{2}$ is due to spontaneous emission from state $|n\rangle$ and to resonant atomic collisions. ${ }^{30}$ These two effects contribute to $T_{2}$ in the following manner:

$$
\frac{1}{T_{2}}=\frac{1}{2 \tau_{s}}+\frac{1}{\tau_{c}},
$$

where $\tau_{s}$ is the spontaneous radiation lifetime for state $|n\rangle$ and $\tau_{c}$ is the time between collisions. Consequently, it is possible to divide the absorption coefficient into the two contributions

$$
\alpha_{0}=\alpha_{s}+\alpha_{c},
$$

where $\alpha_{s}$ describes that part of the absorption due to spontaneous emission and $\alpha_{c}$ describes that part due to collisions. According to several author $\mathrm{s}^{21,31-35}$ the absorption of energy from the $\overrightarrow{\mathrm{E}}_{2}$ pulse due to $\alpha_{s}$ does not excite state $|n\rangle$, but describes a scattering of the $\overrightarrow{\mathrm{E}}_{2}$ beam only. However, the absorption of energy from the $\overrightarrow{\mathrm{E}}_{2}$ pulse due to $\alpha_{c}$ does excite state $|n\rangle$. Since the total attenuation of the pulsed beam was negligible, the number density $N_{n}(t)$ of atoms incoherently excited to the state $|n\rangle$ during the pulse is given by

$$
N_{n}(t)=\alpha_{c} \Re(t),
$$

where $\mathscr{l}(t)$ is the total number of photons per $\mathrm{cm}^{2}$ that have propagated past the atomic volume. This relationship is given in more detail by

$$
N_{n}(t)=\frac{4 \pi \omega_{2} N p_{n 2}^{2}}{c \tau \hbar \Delta \omega^{2}} \frac{c}{4 \pi \hbar \omega_{2}} \int_{0}^{t} \mathscr{S}_{2}^{2}\left(t^{\prime}\right) d t^{\prime} .
$$

In Eq. (7) it has been assumed that $T_{1}$ is long compared to the pulse width. It will be useful to introduce the maximum value $N_{n m}$ of $N_{n}(t)$, defined to be

$$
N_{n m}=\frac{4 \pi \omega_{2} N \phi_{n 2}^{2}}{c \tau_{c} \hbar \Delta \omega^{2}} \frac{c \mathscr{S}_{2 m}^{2} \Delta t}{4 \pi \hbar \omega_{2}},
$$

with

$$
\mathfrak{S}_{2 m}^{2} \Delta t=\int_{0}^{\infty} \mathcal{E}_{2}^{2}\left(t^{\prime}\right) d t^{\prime},
$$

where $\mathcal{E}_{2 m}$ designates the maximum value of $\mathcal{E}_{2}$ and $\Delta t$ is approximately equal to the pulse width. It is instructive to take the ratio

$$
N_{n m}^{*} / N_{n m}=\tau_{c} / 2 \Delta t,
$$

where $N_{n m}^{*}=N_{n}^{*}(t)$ at the peak of the pulse. Clearly, $N_{n m}^{*}$ can be larger than the total incoherent excitation $N_{n m}$ if the pulse width is short compared to the collision time; this is the situation for our experiment, where $N_{n m}^{*} / N_{n m}=12$. Consequently, CE is not a small effect, and the peak coherent excitation can be much larger than the total incoherent excitation. Coherent excitation dominates the energy transfer between the electromagnetic wave and the atomic system, and, as shown in Ref. 13, is responsible for slow group velocities.

\section{ADIABATIC-STATES THEORY}

In order to interpret the meaning of the coherent and incoherent excitation discussed in Sec. II, we introduce the adiabatic states. The physical idea is that the presence of the $\overrightarrow{\mathrm{E}}_{2}$ pulse changes the atomic Hamiltonian and that the adiabatic states are the stationary states ${ }^{22-24}$ of this new Hamiltonian. These states have been used previously to discuss Stark effects in rapidly varying fields, ${ }^{25}$ and for spin resonance their importance has been experimentally demonstrated. ${ }^{26,27}$ In the optical regime these states are used in the study of the two-photon resonance ${ }^{16-19}$ in the description of resonance scattering and fluorescence,$^{28}$ and in the explanation of parametric mixing processes. ${ }^{36}$ An alternative approach to this problem is to use a full quantum-mechanical treatment where the applied field is described in terms of photons. ${ }^{37-39}$ The resulting states of the "dressed" atom ${ }^{38,39}$ appear to be the same as the adiabatic states.

Because the intensity of the probing beam $\overrightarrow{\mathrm{E}}_{1}$ is considered to be vanishingly small, the probing beam monitors the changes in the atomic system caused by the presence of the $\overrightarrow{\mathrm{E}}_{2}$ pulse. Thus the $\vec{E}_{1}$ beam allows for transient atomic spectroscopy of the atom which is strongly perturbed by the $\overrightarrow{\mathrm{E}}_{2}$ pulse. The resonances obtained for the $\vec{E}_{1}$ beam 
correspond to transitions between the atomic states when the atom is in the strong $\overrightarrow{\mathrm{E}}_{2}$ pulse; these transitions occur only between the adiabatic states.

\section{A. Adiabatic states}

The adiabatic states will now be calculated following the formalism developed in Ref. 19, where their importance in describing the two-photon resonance was demonstrated. The Hamiltonian describing the atom and its interaction with the applied light is of the form

$$
\mathfrak{H}=\mathcal{H}_{0}-\overrightarrow{\mathrm{p}} \cdot \overrightarrow{\mathrm{E}},
$$

where $\mathfrak{H}_{0}$ is the atomic Hamiltonian in the absence of the light, $\overrightarrow{\mathrm{p}}$ is the electric-dipole-moment operator, and $\overrightarrow{\mathrm{E}}$ is the total electric field of the light. $\overrightarrow{\mathrm{E}}$ is given by

$$
\overrightarrow{\mathrm{E}}=\overrightarrow{\mathrm{E}}_{1}+\overrightarrow{\mathrm{E}}_{2}
$$

with

$$
\begin{aligned}
& \overrightarrow{\mathrm{E}}_{1}=\mathcal{E}_{1}\left(\hat{x} \cos \omega_{1} t+\hat{y} \sin \omega_{1} t\right), \\
& \overrightarrow{\mathrm{E}}_{2}=\mathcal{S}_{2}\left(\hat{x} \cos \omega_{2} t+\hat{y} \sin \omega_{2} t\right) .
\end{aligned}
$$

The eigenstates of $\mathfrak{H}_{0}$ are designated by $|1\rangle,|2\rangle$, and $|n\rangle$, and are shown in Fig. 1. The corresponding adiabatic states $|1\rangle^{\prime},|2\rangle^{\prime}$, and $|n\rangle^{\prime}$ are the stationary states of $\mathcal{H}$. These adiabatic states evolve continuously from the original eigenstates as the time-dependent perturbation $\overrightarrow{\mathrm{p}} \cdot \overrightarrow{\mathrm{E}}$ is applied. Following Eqs. (24)-(26) of Ref. 19, the adiabatic states are equal to

$$
|i\rangle^{\prime}=\sum_{n} U_{n i}^{-1}|n\rangle,
$$

where $U$ is the unitary operator that transforms from the original basis of the eigenstates of $\mathcal{H}_{0}$ to the new basis of the adiabatic states. For the experiment considered here the intensity of the cw probing beam is considered to be vanishingly small. Consequently, the unitary transformation $U$ is given by

$$
U=\left[\begin{array}{ccc}
1 & 0 & 0 \\
0 & \cos \frac{1}{2} \theta^{\prime} & -\left(\sin \frac{1}{2} \theta^{\prime}\right) e^{-i \omega_{2} t} \\
0 & \left(\sin \frac{1}{2} \theta^{\prime}\right) e^{+i \omega_{2} t} & \cos \frac{1}{2} \theta^{\prime}
\end{array}\right]
$$

The top row in Eq. (13) displays $U_{11}, U_{1 n}$, and $U_{12}$, respectively. Thus with $U^{-1}=U^{\dagger}$ the adiabatic states are obtained from Eqs. (12) and (13) as

$$
\begin{aligned}
& |1\rangle^{\prime}=|1\rangle, \\
& |n\rangle^{\prime}=-\left(\sin \frac{1}{2} \theta^{\prime}\right) e^{i \omega_{2} t}|2\rangle+\left(\cos \frac{1}{2} \theta^{\prime}\right)|n\rangle, \\
& |2\rangle^{\prime}=\left(\cos \frac{1}{2} \theta^{\prime}\right)|2\rangle+\left(\sin \frac{1}{2} \theta^{\prime}\right) e^{-i \omega_{2} t}|n\rangle .
\end{aligned}
$$

In terms of the vector model describing the $\Omega_{n 2}$ transition, the adiabatic excited state $|n\rangle^{\prime}$ corresponds to the pseudomoment antiparallel to the effective field vector, while the adiabatic ground state $|2\rangle^{\prime}$ corresponds to the pseudomoment parallel to the effective field vector. Therefore the adiabatic following approximation shown in Fig. 2 (b) is equivalent to the atom remaining in the adiabatic ground state.

\section{B. Transitions between adiabatic states}

While the pulsed field is present, the weak cw laser probing beam can cause transitions only between the adiabatic states. ${ }^{22-28}$ Furthermore, because the field $\vec{E}_{2}$ is applied adiabatically, according to the adiabatic theorem of quantum mechanics, ${ }^{23}$ all of the atoms remain in the adiabatic ground state $|2\rangle^{\prime}$ (in the absence of relaxation processes). Thus the wave function for the atomic system during the pulse is given by

$$
\Psi_{2}^{\prime}=|2\rangle^{\prime} e^{-\Omega_{2}^{\prime} t}
$$

where

$$
\hbar \Omega_{2}^{\prime}=\hbar \Omega_{2}+\Delta E_{2},
$$

and the optical Stark shift is given by $\Delta E_{2}$ [see Eq. (B4b) of Ref. 19]. For completeness, primes on all of the energies $\Omega_{1}^{\prime}, \Omega_{n}^{\prime}$, and $\Omega_{2}^{\prime}$ will now be introduced in the formalism to signify the Starkshifted values, even though for this experiment the optical Stark shift is much less than the inhomogeneous linewidths of any of the considered transitions and could be neglected without changing any of the conclusions.

Following the procedure of Townes and Schawlow, ${ }^{25}$ we can calculate the resonant frequency for absorption of the $\vec{E}_{1}$ probing beam due to coherent excitation. The absorption is due to the transition from the adiabatic ground state $|2\rangle^{\prime}$ to the adiabatic excited state $|1\rangle^{\prime}$, where the wave function for $|1\rangle^{\prime}$ is equal to

$$
\Psi_{1}^{\prime}=|1\rangle^{\prime} e^{-i \Omega} \dot{1}^{\prime} .
$$

We take the matrix element of the interaction term $\overrightarrow{\mathrm{p}} \cdot \overrightarrow{\mathrm{E}}_{1}$ between the adiabatic ground and excited states, where

$$
\left\langle\Psi_{2}^{\prime}\left|\overrightarrow{\mathrm{p}} \cdot \overrightarrow{\mathrm{E}}_{1}\right| \Psi_{1}^{\prime}\right\rangle={ }^{\prime}\left\langle 2\left|\overrightarrow{\mathrm{p}} \cdot \overrightarrow{\mathrm{E}}_{1}\right| 1\right\rangle^{\prime} e^{-i \Omega 1_{2} t}
$$

Using Eqs. (11b) and (14), Eq. (B2a) of Ref. 19, and remembering that $p_{12}=0$, we recast Eq. (18) into the form

$$
\begin{aligned}
\left\langle\Psi_{2}^{\prime}\left|\overrightarrow{\mathrm{p}} \cdot \overrightarrow{\mathrm{E}}_{1}\right| \Psi_{1}^{\prime}\right\rangle= & \left(\sin \frac{1}{2} \theta^{\prime}\right)\left(p_{n 1} \mathcal{E}_{1} / \sqrt{2}\right) \\
& \times e^{-i\left(\Omega_{12}^{\prime}-\omega_{1}-\omega_{2}\right) t}
\end{aligned}
$$

Thus it is seen from Eq. (19) that the resonant frequency for the transition between $|2\rangle^{\prime}$ and $|1\rangle^{\prime}$ with respect to the probing beam is the usual two- 
photon resonance condition $\omega_{1}+\omega_{2}=\Omega_{12}^{\prime}$. Note that the transition is allowed only by the mixture of state $|n\rangle$ in the adiabatic ground state $|2\rangle^{\prime}$, and that this coherent excitation of state $|n\rangle$ appears to have the shifted energy $\omega_{2}+\Omega_{2}^{\prime}$, independent of $\Omega_{n}$. These considerations are the topic of Sec. IV.

The situation is quite different for the incoherently absorbed light. There an absorption of an $\omega_{2}$ photon corresponds to the transition $|2\rangle^{\prime}-|n\rangle^{\prime}$. Then, an $\omega_{1}$ photon can be subsequently absorbed by the atom in the first adiabatic excited state $|n\rangle^{\prime}$. This argument can be made more precise by calculating the matrix element of $\overrightarrow{\mathrm{p}} \cdot \overrightarrow{\mathrm{E}}_{1}$ between $|n\rangle^{\prime}$ and the highest excited state $|1\rangle^{\prime}$,

$$
\left\langle\Psi_{n}^{\prime}\left|\overrightarrow{\mathrm{p}} \cdot \overrightarrow{\mathrm{E}}_{1}\right| \Psi_{1}^{\prime}\right\rangle=\left\langle n\left|\overrightarrow{\mathrm{p}} \cdot \overrightarrow{\mathrm{E}}_{1}\right| 1\right\rangle^{\prime} e^{-i \Omega_{1}^{\prime} t}
$$

Again, we can recast Eq. (20) in the form

$$
\begin{aligned}
\left\langle\Psi_{n}^{\prime}\left|\overrightarrow{\mathrm{p}} \cdot \overrightarrow{\mathrm{E}}_{1}\right| \Psi_{1}^{\prime}\right\rangle= & \left(\cos \frac{1}{2} \theta^{\prime}\right)\left(p_{n 1} \mathcal{E}_{1} / \sqrt{2}\right) \\
& \times e^{-i\left(\Omega_{1}^{\prime}-\omega_{1}\right) t} .
\end{aligned}
$$

Equation (21) shows a resonance when $\omega_{1}=\Omega_{1 n}^{\prime}$. This is in agreement with the above discussion and shows that the probing beam monitors the excitation of state $|n\rangle^{\prime}$. An alternative treatment based on the density-matrix approach is presented in Ref. 40, where the same conclusion is reached.

For completeness, a closely related situation in spin resonance will be briefly described. For the case of double resonance for nuclear spins, Anderson $^{41}$ considers the situation of simultaneous application of a strong radio-frequency field with frequency $\omega_{1}$ and a weak probing field with frequency $\omega_{2}$. He shows that for the probing field there are two resonances, $\omega_{2}=\omega_{1} \pm \gamma H_{\text {eff }}$, where $\gamma H_{\text {eff }}=\left[\left(\omega_{A}-\omega_{1}\right)^{2}+\left(\gamma H_{1}\right)^{2}\right]^{1 / 2}, \omega_{A}$ designates the resonance frequency, $\gamma$ designates the gyromagnetic ratio, and $H_{1}$ designates the magnetic field of the strong field. This result corresponds to the spins precessing about the effective field in the rotating frame with the precession frequency $\gamma H_{\text {eff }}$. When transformed back to the laboratory frame, the free precession contains the two frequencies $\omega_{1} \pm \gamma H_{\mathrm{cff}}$. The angular frequency $\omega_{2}$ of the weak radio-frequency field must be made equal to one of these frequencies in order to produce resonance.

\section{COHERENT AND INCOHERENT EXCITATION}

We can now understand the meaning of the coherent excitation calculated by the vector model in Sec. II. When the adiabatic following approximation is valid, CE describes the contribution of the unperturbed excited state $|n\rangle$ to the adiabatic ground state $|2\rangle^{\prime}$. This conclusion is contained in Eq. (14c), which is of the form

$$
|2\rangle^{\prime}=a_{2}|2\rangle+a_{n}|n\rangle \text {. }
$$

Thus CE is obtained as

$$
N_{n}^{*}(t)=N a_{n}^{*} a_{n}=N \sin ^{2} \frac{1}{2} \theta^{\prime},
$$

which is exactly the same as Eq. (1) calculated by the vector model. However, as shown in Sec. III and in agreement with experiment, $\mathrm{CE}$ does not behave as an ordinary atomic population in state $|n\rangle$. Some insight into this can be gained by rewriting the two-photon resonance condition of Eq. (19) as follows:

$$
\Omega_{12}^{\prime}-\left(\omega_{1}+\omega_{2}\right)=\left[\Omega_{1}^{\prime}-\left(\omega_{2}+\Omega_{2}^{\prime}\right)\right]-\omega_{1} .
$$

From Eq. (24) it can be seen that while the coherent excitation appears to be a population in state $|n\rangle$, the energy corresponding to the coherent excitation is not $\Omega_{n}$, but is $\omega_{2}+\Omega_{2}^{\prime}$. With this change in energy the coherent excitation behaves as any other excitation.

Thus we can see that the energy density $U_{c}$ of the coherent excitation is equal to the number density $N_{n}^{*}(t)$ multiplied by the adiabatic excited-state energy $\hbar\left(\omega_{2}+\Omega_{2}^{\prime}\right)$ and minus the adiabatic ground-state energy $\hbar \Omega_{2}^{\prime}$, i.e.,

$$
U_{c}=\hbar \omega_{2} N_{n}^{*}(t)
$$

This conclusion is in agreement with the work of Hahn and Diels, ${ }^{7}$ where energy balance for off-resonant pulse propagation was studied.

Now, using the conclusion that $N_{n}^{*}(t)$ behaves as an ordinary excitation with the shifted energy $\hbar\left(\omega_{2}\right.$ $\left.+\Omega_{2}^{\prime}\right)$, we can calculate the dielectric constant $\epsilon_{p}^{*}$ and absorption coefficient $\alpha_{p}^{*}$ caused by $N_{n}^{*}(t)$ and measured by the $\mathrm{cw}$ probing beam. We will then relate $\epsilon_{p}^{*}$ and $\alpha_{p}^{*}$ to the equivalent results for the two-photon resonance, and they will be seen to be identical. Thus the near-resonant dielectric constant $\epsilon_{p}^{*}$ for the $\vec{E}_{1}$ probing beam is obtained from the corresponding single-photon result ${ }^{5,13}$ by treating $N_{n}^{*}(t)$ as an ordinary atomic population with the transition frequency $\Omega_{1}^{\prime}-\left(\omega_{2}+\Omega_{2}^{\prime}\right)$,

$$
\epsilon_{p}^{*}=1+\frac{4 \pi N_{n}^{*}(t) p_{n 1}^{2}}{\hbar\left[\Omega_{1}^{\prime}-\left(\omega_{2}+\Omega_{2}^{\prime}\right)-\omega_{1}\right]} .
$$

To show the connection between $\mathrm{CE}$ and the twophoton resonance, Eq. (26) can be rewritten in the form ${ }^{40}$

$$
\epsilon_{p}^{*}=1+\frac{2 \pi N p_{n 2}^{2} p_{n 1}^{2} \mathcal{E}_{2}^{2}}{\hbar^{3}(\Delta \omega)^{2} \delta^{\prime}}
$$

with

$$
\delta^{\prime}=\Omega_{1}^{\prime}-\left(\omega_{2}+\Omega_{2}^{\prime}\right)-\omega_{1}=\Omega_{12}^{\prime}-\left(\omega_{1}+\omega_{2}\right),
$$

and where use was made of Eq. (2). Equation (27) is in a convenient form to compare with the nonlinear polarization associated with the two-photon resonance. From Eq. (B13b) of Ref. 19 we obtain 


$$
\epsilon_{p}^{*}=1+2 \pi \chi^{(3)} \mathcal{E}_{2}^{2},
$$

where the nonlinear coefficient $\chi^{(3)}$ reduces to

$$
\chi^{(3)}=p_{n 2}^{2} p_{n 1}^{2} / \hbar^{3}(\Delta \omega)^{2} \delta^{\prime} .
$$

Thus the dielectric constant $\epsilon_{p}^{*}$ for the $\overrightarrow{\mathrm{E}}_{1}$ beam due to $\mathrm{CE}$ is the same as that derived from the nonlinear polarization due to the two-photon resonance. The concept of coherent excitation provides an alternative way of understanding the multiphoton processes.

Equations (27) and (28) show the important result that it is possible to control the dielectric constant of one light beam $\left(\vec{E}_{1}\right.$ beam) by a second switching beam ( $\overrightarrow{\mathrm{E}}_{2}$ pulse) and to base a modulator on this principle. This concept was introduced by Armstrong and Grischkowsky, ${ }^{42}$ where at that time the connection with the two-photon resonance was not realized. Recently, Liao and Bjorklund ${ }^{43}$ have experimentally demonstrated this effect and explained the relationship to the two photon resonance.

Also, the absorption coefficient $\alpha_{p}^{*}$ for the $\overrightarrow{\mathrm{E}}_{1}$ beam is obtained from the single-photon result, and when the frequency $\omega_{1}$ is within the Dopplerbroadened line, $\alpha_{p}^{*}$ is equal to

$$
\begin{aligned}
\alpha_{p}^{*}= & \frac{16 \pi^{3} N_{n}^{*}(t) p_{n 1}^{2}(\ln 2 / \pi)^{1 / 2}}{\lambda_{1} \hbar \Delta \omega_{D}} \\
& \times \exp \left[-\left(\frac{2 \delta^{\prime}}{\Delta \omega_{D}}\right)^{2} \ln 2\right] .
\end{aligned}
$$

Using Eq. (2) for $N_{n}^{*}(t)$, we can recast Eq. (30) into the more familiar form for the two-photon resonance, ${ }^{44}$

$$
\begin{aligned}
\alpha_{p}^{*}= & \frac{8 \pi^{3} N p_{n 2}^{2} p_{n 1}^{2} \mathcal{E}_{2}^{2}(\ln 2 / \pi)^{1 / 2}}{\lambda_{1} \hbar^{3}(\Delta \omega)^{2} \Delta \omega_{D}} \\
& \times \exp \left[-\left(\frac{2 \delta^{\prime}}{\Delta \omega_{D}}\right)^{2} \ln 2\right] .
\end{aligned}
$$

The analysis for the incoherent excitation $N_{n}(t)$ is similar. However, for $N_{n}(t)$ the energy of state $|n\rangle^{\prime}$ is equal to $\Omega_{n}^{\prime}$. Thus the energy density $U_{n}$ of the incoherent excitation is given simply by

$$
U_{n}=\hbar \Omega_{n 2}^{\prime} N_{n}(t) .
$$

For incoherent excitation we obtain for the nearresonant dielectric constant $\epsilon_{p}$ of the probing beam

$$
\epsilon_{p}=1+\frac{4 \pi N_{n}(t) p_{n 1}^{2}}{\hbar\left(\Omega_{1 n}^{\prime}-\omega_{1}\right)} .
$$

Finally, the absorption coefficent $\alpha_{p}$ for the $\vec{E}_{1}$ beam, when $\omega_{1}$ is tuned within the Doppler width of the $\Omega_{1 n}^{\prime}$ transition, is given by


FIG. 3. Schematic diagram showing the simultaneous coherent excitation $N_{n}^{*}(t)$ and incoherent excitation $N_{n}(t)$ produced by the $\overrightarrow{\mathrm{E}}_{2}$ pulse. The appropriate tuning frequency $\omega_{1}$ of the $\overrightarrow{\mathrm{E}}_{1}$ probing beam is shown for monitoring either $N_{n}^{*}(t)$ or $N_{n}(t)$.

$$
\begin{aligned}
\alpha_{p}= & \frac{16 \pi^{3} N_{n}(t) p_{n 1}^{2}(\ln 2 / \pi)^{1 / 2}}{\lambda_{1} \hbar \Delta \omega_{D}} \\
& \times \exp \left[-\left(\frac{2\left(\Omega_{1 n}^{\prime}-\omega_{1}\right)}{\Delta \omega_{D}}\right)^{2} \ln 2\right]
\end{aligned}
$$

where $\Delta \omega_{D}$ designates the Doppler width full width at half-maximum of the single-photon transition.

As shown in Fig. 3, the simultaneous coherent excitation and the incoherent excitation differ in three important respects. First, the relative magnitudes of the coherent and incoherent excitation can be quite different, depending on the experimental conditions. For our example to follow, the coherent excitation is more than 10 times the incoherent excitation. Second, the excitation energy $\omega_{2}+\Omega_{2}^{\prime}$ of coherent excitation is determined by the driving frequency $\omega_{2}$, while the excitation energy $\Omega_{n}^{\prime}$ of the incoherent excitation is given by that of the excited state $|n\rangle^{\prime}$. Third, the coherent excitation is proportional to the pulse intensity, while for relaxation times long compared to the pulse width the incoherent excitation is proportional to the integral of the pulse intensity. Consequently, the cw probing beam can be tuned to monitor either the coherent excitation or the incoherent excitation, as shown in Fig. 3. This separation is possible because the frequency offset $\Delta \omega$ is relatively large compared to the inhomogeneous linewidth and because the $\overrightarrow{\mathrm{E}}_{2}$ pulse changes adiabatically. When $\omega_{2} \approx \Omega_{n 2}$ this experimental distinction is no longer possible. 


\section{EXPERIMENT}

The double-resonance experiment employed a relatively strong $\overrightarrow{\mathrm{E}}_{2}$ dye-laser pulse and a weak $\overrightarrow{\mathrm{E}}_{1} \mathrm{cw}$ dye-laser probing beam. The two beams were circularly polarized and propagated through a $100-\mathrm{cm} \mathrm{Rb}$-vapor cell and along a tunable magnetic field. The driving frequency $\omega_{2}$ was tuned approximately $1 \mathrm{~cm}^{-1}$ away (on the low-frequency side) from the resonant frequency $\Omega_{n 2}(7948 \AA)$ of the $5 S_{1 / 2}-5 P_{1 / 2}$ transition of $\mathrm{Rb}$. The probing frequency $\omega_{1}$ was tuned either to the single-photon resonance $\Omega_{1 n}(6206 \AA)$ of the $5 P_{1 / 2} \longrightarrow 6 D_{3 / 2}$ transition or to the two-photon resonance $\omega_{1}+\omega_{2}=\Omega_{12}$ of the $5 S_{1 / 2} \longrightarrow 6 D_{3 / 2}$ transition. The rough frequency tuning was done by tuning $\omega_{1}$ and $\omega_{2}$, while the fine tuning was done by tuning the transition frequencies $\Omega_{n_{2}}$ and $\Omega_{1 n}$ by the magnetic field. During the passage of the $\overrightarrow{\mathrm{E}}_{2}$ pulse through the cell, the output $\mathrm{cw}$ beam was monitored as a function of time.

The dye laser ${ }^{5,13}$ (DTTC in methanol) was longitudinally pumped by a $Q$-switched ruby laser and produced a beam with a peak power of the order of $500 \mathrm{~W}$ and a linewidth of approximately 0.005 $\mathrm{cm}^{-1}$. The $\overrightarrow{\mathrm{E}}_{1}$ probing beam was from a Coherent Radiation Model $490 \mathrm{cw}$ dye laser, which with internal etalons had a linewidth of less than 0.003 $\mathrm{cm}^{-1}$. The Pyrex glass cell was filled by doubly distilling an excess of $\mathrm{Rb}$ into the cell and then sealing under vacuum. The atomic number density $N$ of $\mathrm{Rb}$ is obtained from the cell temperature and the vapor-pressure curve of $\mathrm{Rb}$.

An important experimental requirement for the measurement of $N_{n}(t)$ is that the cw beam not saturate the $\Omega_{1 n}$ transition during the excitation pulse. A rough statement of this condition is that the angle given by the Rabi precession frequency multiplied by the pulse width should be small compared to unity, i.e., $\sqrt{2} p_{n 1} \mathcal{E}_{1} \Delta t / \hbar \ll 1$. For Fig. 4, this angle was $0.07 \mathrm{rad}$, and the condition was well satisfied.

The experimental measurement of the incoherent excitation $N_{n}(t)$ is shown in Fig. 4, where $\omega_{1}=\Omega_{1 n}$. The input $\overrightarrow{\mathrm{E}}_{2}$ pulse had a peak intensity of $200 \mathrm{~W} / \mathrm{cm}^{2}$, a pulse width of $4 \mathrm{nsec}$, and was unchanged by passage through the cell. The transient absorption of the weak $\left(15 \mathrm{~mW} / \mathrm{cm}^{2}\right) \mathrm{cw} \overrightarrow{\mathrm{E}}_{1}$ probing beam is displayed in Fig. 4(c), where the top trace is the zero-intensity baseline and the lower trace is the negative signal due to the $\mathrm{cw}$ beam. Here we see that the absorption follows the integral of the pulse intensity. The absorption still persists after the passage of the pulse, because of the severe radiative trapping which lengthens the lifetime of the excitation to several hundred nsec. For the experimental conditions of
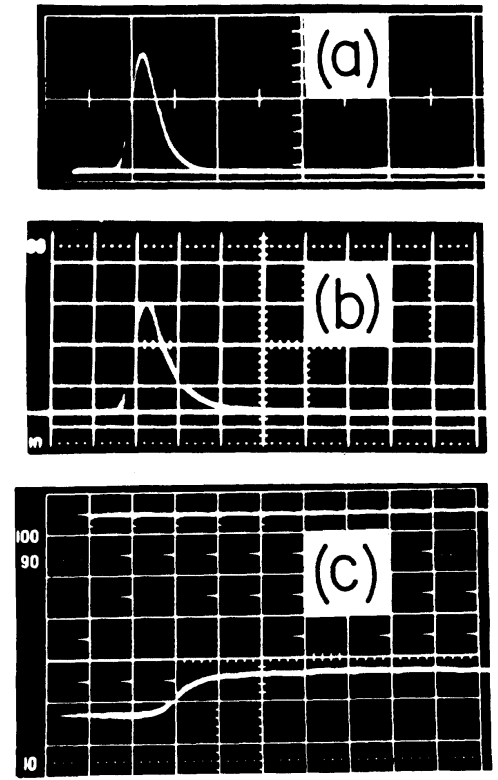

FIG. 4. Experimental measurement of the incoherent excitation $N_{n}(t)$. (a) Input $\vec{E}_{2}$ pulse to the Rb cell with the peak intensity of $200 \mathrm{~W} / \mathrm{cm}^{2}$ and the frequency offset $\Delta \omega=0.8 \mathrm{~cm}^{-1}$. The pulse was measured with an ITT biplanar photodiode and Tektronix 519 oscilloscope. Sweep speed is 10 nsec per large division. (b) Corresponding output pulse measured with an ITT biplanar photodiode and a Tektronix 7904 oscilloscope. Sweep speed is 5 nsec per large division. (c) Transient absorption of the weak $\overrightarrow{\mathrm{E}}_{1}$ probing beam tuned to the singlephoton resonance $\omega_{1}=\Omega_{1 n}$. Top trace is the zero-intensity baseline. Bottom trace is the cw signal, which shows transient absorption due only to $N_{n}(t)$. The signal was measured with a Spectra-Physics 403 photodiode and a Tektronix 7904 oscilloscope. Sweep speed is 5 nsec per large division.

Fig. 4 and as indicated in Fig. 3 the peak coherent excitation $N_{n m}^{*}$ is very much larger than the peak incoherent excitation $N_{n m}$. The ratio $N_{n m}^{*} / N_{n m}=12$ is obtained from Eq. (9) together with the collision time $\tau_{c}=100 \mathrm{nsec}$ (see the Appendix) and the pulse width $\Delta t=4 \mathrm{nsec}$ from Fig. 4 . However, only $N_{n}(t)$ is observed. This striking separation of these two effects is due to the frequency offset $\Delta \omega=0.8$ $\mathrm{cm}^{-1}$, which is much larger than the inhomogeneous linewidth of the $\Omega_{12}$ transition. For the on-resonance case $(\Delta \omega=0)$ both the coherent and the incoherent excitation would be monitored simultaneously, and the results would be much more difficult to interpret. Figure 4 also shows, except for the absorption due to $\alpha_{c}$, how well the $\mathrm{Rb}$ atoms remain in the adiabatic ground state $|2\rangle^{\prime}$ throughout the pulse. If this were not the case, a marked difference in the transient absorption would be expected due to the large magnitude of $N_{n}^{*}(t)$. Equivalently, this result shows the validity of the 
adiabatic following approximation to describe offresonant coherent effects.

It is possible to compare the observed peak absorption with theory by using the on-resonance form of Eq. (34), with $N_{n}(t)$ replaced by $N_{n m}$ given by Eq. (8a). Thus the peak absorption coefficient $\alpha_{p m}$ on line center is given by

$$
\alpha_{p m}=\frac{16 \pi^{3} p_{n 1}^{2}(\ln 2 / \pi)^{1 / 2}}{\lambda_{1} \hbar \Delta \omega_{D}} \frac{4 \pi \omega_{2} N p_{n 2}^{2}}{c \tau_{c} \hbar \Delta \omega^{2}} \frac{c \mathcal{E}_{2 m}^{2} \Delta i}{4 \pi \hbar \omega_{2}} .
$$

The cell temperature was $140^{\circ} \mathrm{C}$ corresponding to the effective ${ }^{45} \mathrm{Rb}$ atomic number density $N=3 \times$ $\times 10^{13} \mathrm{~cm}^{-3}$; the frequency offset of the $\overrightarrow{\mathrm{E}}_{2}$ pulse was $\Delta \omega / 2 \pi c=0.8 \mathrm{~cm}^{-1}$; the pulse width was $\Delta t=4$ nsec; the effective Doppler width including the hyperfine splitting of the $5 P_{1 / 2}$ state was $\Delta \omega_{D} /$ $2 \pi c=0.04 \mathrm{~cm}^{-1}$; as shown in the Appendix, the measured collision time was $\tau_{c}=100 \mathrm{nsec}$; the matrix element of the electric dipole moment for circularly polarized light between the $5 S_{1 / 2}$ and $5 P_{1 / 2}$ states is $p_{n 2}=6.16 \times 10^{-18} \mathrm{esu}$; and the matrix element for circularly polarized light between the $5 P_{1 / 2}$ and the $6 D_{3 / 2}$ states is $p_{n 1}=1.55$ $\times 10^{-18}$ esu. $^{46}$

Evaluating Eq. (35) with the above parameters we obtain $\alpha_{p m}=0.17 \times 10^{-2} \mathrm{~cm}^{-1}$, compared with the experimental value $\alpha_{p m}=0.2 \times 10^{-2} \mathrm{~cm}^{-1}$. This very good agreement is probably somewhat fortuitous, considering the large number of parameters in Eq. (35). However, the agreement does provide an important substantiation of our picture of the absorption process and tentatively confirms that $N_{n}(t)$ is due only to collision processes. If $N_{n}(t)$ were due to both collisions and spontaneous emission, $\tau_{c}$ in Eq. (35) would be replaced by $T_{2}$ $=36 \mathrm{nsec}$ (see the Appendix) and the calculated absorption coefficient would be $\alpha_{p m}=0.5 \times 10^{-2}$ $\mathrm{cm}^{-1}$. It is interesting to note that the time dependence of $N_{n}(t)$ is faster than any relaxation time associated with the system, and yet Eq. (35), which is a steady-state result, agrees with experiment. This situation has been considered by Crisp, ${ }^{47}$ and our results agree with his conclusions.

The experimental measurement of the coherent excitation $N_{n}^{*}(t)$ (two-photon absorption) is shown in Fig. 5 , where $\omega_{1}+\omega_{2}=\Omega_{12}$. The input $\overrightarrow{\mathrm{E}}_{2}$ pulse had a peak intensity of $500 \mathrm{~W} / \mathrm{cm}^{2}$ and was unchanged by passage through the cell. The transient absorption of the weak cw probing beam is displayed in Fig. 5(b), where the top trace is the zero-intensity baseline and the lower trace is the negative signal due to the $\mathrm{cw}$ beam. Here, we see that as expected from the discussion of coherent excitation the absorption follows the intensity of
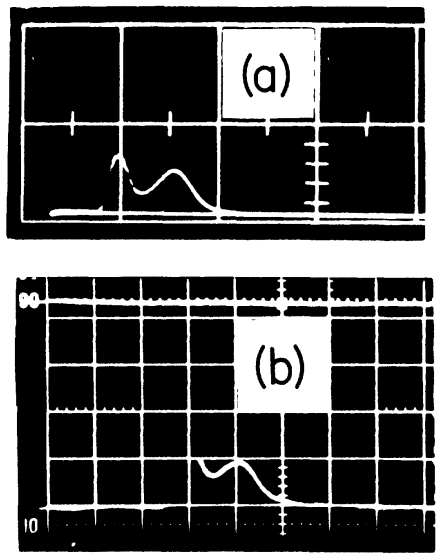

FIG. 5. Experimental measurement of the coherent excitation $N_{n}^{*}(t)$. (a) Input pulse to the Rb cell with the peak intensity of $500 \mathrm{~W} / \mathrm{cm}^{2}$ and the frequency offset $\Delta \omega$ $=0.6 \mathrm{~cm}^{-1}$. The pulse was measured with an ITT biplanar photodiode and a Tektronix 519 oscilloscope. Sweep speed is 10 nsec per large division. (b) Transient absorption of the weak $\overrightarrow{\mathrm{E}}_{1}$ probing beam tuned to the two-photon resonance $\omega_{1}+\omega_{2}=\Omega_{12}$. Top trace is the zero-intensity baseline. Bottom trace is the cw signal which shows transient absorption due only to $N_{n}^{*}(t)$. The signal was measured with a Spectra-Physics 403 photodiode and a Tektronix 7904 oscilloscope. Sweep speed is $5 \mathrm{nsec}$ per large division.

the input pulse, and after passage of the pulse the absorption vanishes. The large magnitude of the observed two-photon absorption is at first sight surprising, since most two-photon resonance studies ${ }^{48-54}$ have monitored fluorescence from the excited state because the absorption was so small. Compared to this earlier work, the large twophoton absorption absorption obtained here is due to the following: (1) relatively strong $\overrightarrow{\mathrm{E}}_{2}$ pulse, (2) resonant enhancement (small $\Delta \omega$ ), (3) fully allowed transitions, and (4) long path lengths. While the fluorescence technique is very sensitive, its time response is limited by the lifetime of the excited state. In contrast, the time response of of our direct measurement is limited only by the detector rise times, and we have better than 1 nsec resolution.

It is important to note that the nanosecond structure of the pulse is short compared to any relaxation time of the system. Consequently, the observed absorption cannot be described by steadystate theory, and transient effects must be considered. Crisp ${ }^{47}$ discusses this case for the single-photon problem, and he shows that the ordinary steady-state absorption coefficient can be used. His procedure can be directly extended to the two-photon resonance using the two-photon vector model, ${ }^{19}$ and the same conclusion is obtained, namely, that the ordinary steady-state 
two-photon absorption coefficient can be used.

It is possible to compare the peak observed absorption with theory by using the on-resonance form of Eq. (31) given below:

$$
\alpha_{p m}^{*}=\frac{8 \pi^{3} N p_{n 2}^{2} p_{n 1}^{2} \mathcal{E}_{2 m}^{2}(\ln 2 / \pi)^{1 / 2}}{\lambda_{1} \hbar^{3}(\Delta \omega)^{2} \Delta \omega_{D}}
$$

For Eq. (36), the frequency offset of the $\vec{E}_{2}$ pulse was $\Delta \omega / 2 \pi c=0.6 \mathrm{~cm}^{-1}$; the cell temperature was $125^{\circ} \mathrm{C}$ corresponding to the effective $e^{45}$ atomic number density $N=1.3 \times 10^{13} \mathrm{~cm}^{-3}$; the effective Doppler width including the hyperfine splitting of the $5 S_{1 / 2}$ ground state was $\Delta \omega_{D} / 2 \pi c=0.2 \mathrm{~cm}^{-1}$.

Evaluating Eq. (36) with the above parameters we obtain $\alpha_{p m}^{*}=0.8 \times 10^{-2} \mathrm{~cm}^{-1}$, compared with the experimental value $\alpha_{p m}^{*}=0.3 \times 10^{-2} \mathrm{~cm}^{-1}$. The agreement is somewhat disappointing compared to the measurement of $N_{n}(t)$, but is not unreasonable considering the large number of parameters involved. There were experimental difficulties involving beam overlap and the different group velocities for the $\vec{E}_{2}$ pulse and the $\overrightarrow{\mathrm{E}}_{1}$ probing beam which served to reduce the observed absorption. Also, it was relatively difficult to tune the two frequencies to exact line center. The large magnitude of the observed two-photon absorption demonstrates that under more precise conditions this experimental technique would allow one to accurately measure the matrix element $p_{n 1}$, which is relatively difficult to obtain by other methods.

\section{CONCLUSIONS}

Coherent excitation occurs whenever light interacts with atoms to produce a polarization. The situation studied in this paper, where the frequency $\omega_{2}$ of a relatively strong $\vec{E}_{2}$ pulse is nearresonant to an atomic transition with excited state $|n\rangle$, and transition frequency $\Omega_{n 2}$, is now understood. The energy $U_{c}$ in the atomic system due to CE is equal to $U_{c}=\hbar \omega_{2} N_{n}^{*}(t)$, where $N_{n}^{*}(t)$ is the atomic number density in state $|n\rangle$. This important relationship shows that the frequency associated with $U_{c}$ is not the transition frequency $\Omega_{n 2}$ but is the driving frequency $\omega_{2}$. The number density $N_{n}^{*}(t)$ can be calculated by application of the vector model of coherent optics, and it is shown for the low-intensity regime of linear dispersion theory that $N_{n}^{*}(t)$ is proportional to the intensity of the driving pulse.

Incoherent excitation of state $|n\rangle$ occurs simultaneously and is caused by the same relaxation process responsible for the absorption coefficient. The atomic energy density $U_{n}$ due to the incoherent excitation is equal to $U_{n}=\hbar \Omega_{n 2} N_{n}(t)$, where $N_{n}(t)$ is the number density of atoms incoherently excited to state $|n\rangle$. It is very important to note that the frequency associated with this exciation is the transition frequency $\Omega_{n 2}$. Similarly, $N_{n}(t)$ can be can be obtained from the vector model, and for the situation where the pulse width of the $\vec{E}_{2}$ driving pulse is short compared to all relaxation times of the atomic system, $N_{n}(t)$ is proportional to the time integral of the pulse intensity.

The above conclusions regarding $N_{n}^{*}(t)$ and $N_{n}(t)$ are confirmed by our double-resonance experiment. A very weak $\overrightarrow{\mathrm{E}}_{1}$ probing beam with frequency $\omega_{1}$ monitors either $N_{n}^{*}(t)$ or $N_{n}(t)$ by its absorption caused by transitions to the higher excited state $|1\rangle$, as illustrated in Figs. 1 and 3. For the experimental parameters, $N_{n}^{*}(t)$ is more than 10 times $N_{n}(t)$, and yet either $N_{n}^{*}(t)$ or $N_{n}(t)$ can be monitored separately. As shown in Fig. 4, $N_{n}(t)$ is observed when $\omega_{1}=\Omega_{1 n}$ in the complete absence of interference from $N_{n}^{*}(t)$. This frequency condition for $\omega_{1}$ is due to the frequency $\Omega_{n 2}$ associated with the incoherent excitation energy density $U_{n}=\hbar \Omega_{n 2} N_{n}(t)$. Moreover, as expected, the measured $N_{n}(t)$ is proportional to the integral of the pulse intenstiy. Furthermore, as shown in Fig. 5, when $\omega_{1}$ is tuned to the twophoton resonance $\omega_{1}+\omega_{2}=\Omega_{12}$, only $N_{n}^{*}(t)$ is observed. This frequency condition for $\omega_{1}$ is due to the frequency $\omega_{2}$ associated with the coherent excitation energy density $U_{c}=\hbar \Omega_{2} N_{n}^{*}(t)$. Thus for coherent excitation $N_{n}^{*}(t)$ behaves as an atomic number density in the excited state $|n\rangle$ but with the state $|n\rangle$ having the energy $\hbar\left(\omega_{2}+\Omega_{2}\right)$. As expected, the measured $N_{n}^{*}(t)$ is proportional to the driving pulse intensity. Finally, the measured values of $N_{n}(t)$ and $N_{n}^{*}(t)$ agree well with theory.

All of the above observations and conclusions are contained in the formalism of the adiabatic states. The physical idea is that the presence of the $\vec{E}_{2}$ pulse changes the atomic Hamiltonian and that the adiabatic states are stationary states of this new Hamiltonian. The experimental results dramatically confirm the point of view that the weak $\vec{E}_{1}$ probing beam can cause transitions only between the adiabatic states. This important conclusion establishes the validity of a recent approach $^{19}$ taken to study coherent effects associated with the two-photon resonance. There, the problem of the interaction between the light and the atomic system is recast in terms of the adiabatic states into an effective two-level system and a twophoton vector model is introduced.

\section{ACKNOWLEDGMENTS}

I would like to acknowledge careful readings of this manuscript by J. A. Armstrong, M. M. T. Loy, and J. J. Wynne. Also, the expert technical support of R. J. Bennett was essential to this work. 


\section{APPENDIX: MEASUREMENT OF $T_{2}$}

Figure 6 shows the result of passing a linearly polarized weak $\overrightarrow{\mathrm{E}}_{2}$ pulse through the $\mathrm{Rb}$ cell when the magnetic field is relatively strong. ${ }^{13}$ Here, the linearly polarized input pulse behaves as two equal-amplitude circularly polarized pulses with opposing senses of rotation, designed as the $\sigma^{-}$ and the $\sigma^{+}$pulses, respectively. The magnitude of the magnetic field was such that the frequency offset $\Delta \omega^{-}=\Omega_{n 2}^{-}-\omega_{2}$ for the $\sigma^{-}$components of the Zeeman-split line was much less than the frequency offset $\Delta \omega^{+}=\Omega_{12}^{+}-\omega_{2}$ for the $\sigma^{+}$components. Consequently, the group velocity $v_{g}^{-}$for the $\sigma^{-}$pulse was much less than $v_{g}^{+}$for the $\sigma^{+}$pulse. For Fig. 6 , the difference in the group velocities was so large that the pulses were completely separated after passage through the cell. It is shown below that Fig. 6(b) provides all of the information required to obtain $T_{2}$, the transverse relaxation time of the $\Omega_{n 2}$ transition. One does not need to know dipole moments, number densities, hyperfine splittings, or the magnetic field.

The above conclusion regarding the measurements of $T_{2}$ will now be demonstrated. The group velocities $v_{g}^{-}$and $v_{g}^{+}$are given by $^{13}$
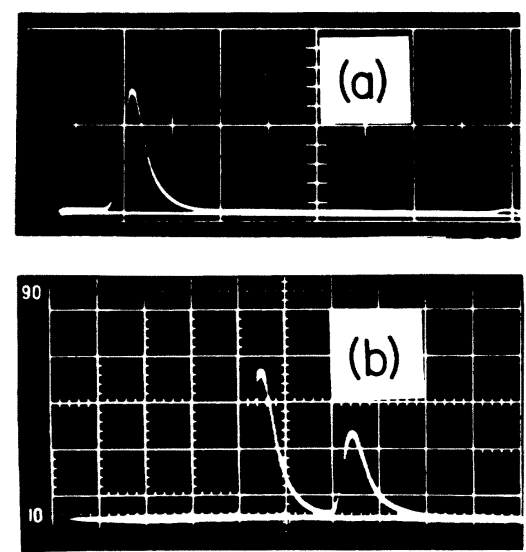

FIG. 6. (a) Input linearly polarized $\overrightarrow{\mathrm{E}}_{2}$ pulse to the $100-\mathrm{cm} \mathrm{Rb}$ cell with the peak intensity of $6 \mathrm{~W} / \mathrm{cm}^{2}$. The pulse was measured with an ITT biplanar photodiode and a Tektronix 519 oscilloscope. Sweep speed is $10 \mathrm{nsec}$ per large division. (b) Resulting two circularly polarized $\sigma^{+}$and $\sigma^{-}$output pulses from the cell. The pulses were monitored with an ITT biplanar photodiode and a Tektronix 7904 oscilloscope. The sweep speed is 5 nsec per large division, giving the measured pulse separation of $9.5 \mathrm{nsec}$, corresponding to the group velocities of $v_{g}^{-}=\frac{1}{4} c$ and $v_{g}^{+}=c / 1.2$ for the $\sigma^{-}$and $\sigma^{+}$pulses, respectively. The $\mathrm{Rb}$ cell temperature was $140{ }^{\circ} \mathrm{C}$ and the cell was in a magnetic field of $9 \mathrm{kG}$. The frequency $\omega_{2}$ of the laser light was $0.4 \mathrm{~cm}^{-1}$ below the center frequency of the $\sigma^{-}$hyperfine components of the Zeemansplit $\Omega_{n 2}$ resonance line and $1.5 \mathrm{~cm}^{-1}$ below the center of the $\sigma^{+}$components.

$$
\begin{aligned}
& \frac{1}{v_{g}^{-}}=\frac{1}{c}+\frac{2 \pi \omega_{2} p_{n 2}^{2}}{c \hbar} \sum_{i} \frac{N_{i}}{\left(\Delta \omega_{i}^{-}\right)^{2}}, \\
& \frac{1}{v_{g}^{+}}=\frac{1}{c}+\frac{2 \pi \omega_{2} p_{n 2}^{2}}{c \hbar} \sum_{j} \frac{N_{j}}{\left(\Delta \omega_{j}^{+}\right)^{2}},
\end{aligned}
$$

where the summation indices $i$ and $j$ refer to the $i$ th and $j$ th hyperfine components of the $\sigma^{-}$and $\sigma^{+}$ transitions, respectively. Similarly, the absorption coefficients $\alpha_{0}^{-}$and $\alpha_{0}^{+}$are equal to

$$
\begin{aligned}
& \alpha_{0}^{-}=\frac{4 \pi \omega_{2} p_{n 2}^{2}}{T_{2} c \hbar} \sum_{i} \frac{N_{i}}{\left(\Delta \omega_{i}^{-}\right)^{2}}, \\
& \alpha_{0}^{+}=\frac{4 \pi \omega_{2} p_{n 2}^{2}}{T_{2} c \hbar} \sum_{j} \frac{N_{j}}{\left(\Delta \omega_{j}^{+}\right)^{2}} .
\end{aligned}
$$

Now, we can calculate the difference $\Delta t_{p}$ in the passage times through the cell of length $l$ for the $\sigma^{-}$and $\sigma^{+}$pulses to be

$$
\Delta t_{p}=l / v_{g}^{-}-l / v_{\boldsymbol{g}}^{+},
$$

or equivalently

$$
\Delta t_{p}=\frac{l 2 \pi \omega_{2} p_{n 2}^{2}}{c \hbar}\left(\sum_{i} \frac{N_{i}}{\left(\Delta \omega_{i}^{-}\right)^{2}}-\sum_{j} \frac{N_{j}}{\left(\Delta \omega_{j}^{+}\right)^{2}}\right) .
$$

Also, the ratio of the intensities $I_{2}^{+}$and $\Gamma_{2}$ of the output $\sigma^{+}$and $\sigma^{-}$pulses is given by

$$
I_{2}^{+} / I_{2}^{-}=e^{l\left(\alpha_{0}^{-}-\alpha_{0}^{+}\right)} .
$$

It is convenient to take the natural logarithm,

$$
\ln \left(I_{2}^{+} / I_{2}^{-}\right)=l\left(\alpha_{0}^{-}-\alpha_{0}^{+}\right),
$$

which can be expanded as

$\ln \left(\frac{I_{2}^{+}}{I_{2}^{-}}\right)=\frac{l 4 \pi \omega_{2} p_{n 2}^{2}}{T_{2} c \hbar}\left(\sum_{i} \frac{N_{i}}{\left(\Delta \omega_{i}^{-}\right)^{2}}-\sum_{j} \frac{N_{j}}{\left(\Delta \omega_{j}^{+}\right)^{2}}\right)$.

We can obtain $T_{2}$ by taking the simple ratio

$$
T_{2}=\frac{2 \Delta t_{p}}{\ln \left(I_{2}^{+} / I_{2}^{-}\right)} .
$$

Thus $T_{2}$ can be obtained directly from measurements of Fig. 6(b), and there is no need for the precise number density and frequency offsets which are frequent sources of error.

From Fig. 6(b) we obtain $\Delta t_{p}=9.5 \mathrm{nsec}$ and $I_{2}^{+} / I_{2}^{-}$ $=1.70$. Then from Eq. (A8), $T_{2}$ is calculated to be 36 nsec. Also, from Eq. (4), with the spontaneous lifetime given by $\tau_{s}=28 \mathrm{nsec}$, we obtain the collision time as $\tau_{c}=100 \mathrm{nsec}$.

The above figure for $\tau_{c}$ can be compared to the predicted value for the $5 S_{1 / 2} \rightarrow 5 P_{1 / 2}$ transition of $\mathrm{Rb}$ from Ref. 30 , determined from the number density as

$$
1 / \tau_{c}=\pi \times 0.8 \times 10^{-7} \mathrm{~N},
$$

where $N$ is the total number density and for our temperature of $140^{\circ} \mathrm{C}, N=6 \times 10^{13}$. From Eq. (A9) we obtain $\tau_{c}=66 \mathrm{nsec}$. 
*Work partially supported by the U. S. Office of Naval Research.

${ }^{1}$ I. D. Abella, N. A. Kurnit, and S. R. Hartmann, Phys. Rev. Lett. 13, 567 (1964); Phys. Rev. 141, 391 (1966)

${ }^{2}$ S. L. McCall and E. L. Hahn, Phys. Rev. Lett. 18, 908 (1967); Phys. Rev. 183, 457 (1969); Phys. Rev. A 2 , 861 (1970).

${ }^{3}$ G. B. Hocker and C. L. Tang, Phys. Rev. Lett. 21, 591 (1968); Phys. Rev. 184, 356 (1969).

${ }^{4}$ R. G. Brewer and R. L. Shoemaker, Phys. Rev. A $\underline{6}$, 2001 (1972)

${ }^{5}$ D. Grischkowsky, Phys. Rev. Lett. 24, 866 (1970); D. Grischkowsky and J. A. Armstrong, Phys. Rev. A $\underline{6}$, 1566 (1972); D. Grischkowsky, in Laser Applications to Optics and Spectroscopy, Vol. II of Physics of Quantum Electronics Series, edited by S. F. Jacobs, M. Sargent III, J. F. Scott, and M. O. Scully (AddisonWesley, Reading, Mass., 1975).

${ }^{6}$ M. M. T. Loy, Phys. Rev. Lett. 32 , 814 (1974).

${ }^{7}$ E. L. Hahn and J. C. Diels, in Laser Spectroscopy, edited by R. G. Brewer and A. Mooradian (Plenum, New York, 1974) p. 323. One of the major themes of this informative paper is energy balance for off-resonance pulse propagation.

${ }^{8} \mathrm{~L}$. Brillouin, Wave Propagation and Group Velocity (Academic, New York, 1960).

${ }^{9}$ A. C. G. Mitchell and M. W. Zemansky, Resonance Radiation and Excited Atoms, 2nd ed. (Cambridge U. P., London, 1961)

${ }^{10}$ E. O. Schulz-DuBois, Proc. IEEE 57, 1748 (1969).

${ }^{11}$ C. G. B. Garrett and D. E. McCumber, Phys. Rev. A 1 , $305(1970)$.

${ }^{12}$ M. D. Crisp, Phys. Rev. A $\underline{4}, 2104$ (1971)

${ }^{13}$ D. Grischkowsky, Phys. Rev . A 7, 2096 (1973).

${ }^{14}$ Recent work on the multilevel problem includes the theory of the Raman echo (Ref. 15), theoretical studies of the two-photon resonance (Refs. 16-19) an exact solution of the three-level problem for two-photon resonance (Ref. 20), and a discussion of the importance of adiabatic excitation of $N_{n}^{*}(t)$ to Raman emission (Ref. 21).

${ }^{15}$ S. R. Hartmann, IEEE J. Quantum Electron. QE-4, 802 (1968)

${ }^{16}$ E. M. Belenov and I. A. Poluektov, Zh. Eksp. Teor. Fiz. 56, 1407 (1969) [Sov. Phys.-JETP 29, 754 (1969)].

${ }^{17}$ M. Takatsuji, Phys. Rev. A $\underline{4}, 808$ (1971); 11, 619 (1975).

${ }^{18}$ D. Grischkowsky and M. M. T. Loy, Phys. Rev. A $\underline{12}$, 1117 (1975).

${ }^{19}$ D. Grischkowsky, M. M. T. Loy, and P. F. Liao, Phys. Rev. A 12, 2514 (1975).

${ }^{20}$ R. G. Brewer and E. L. Hahn, Phys. Rev. A 11, 1641 (1975).

${ }^{21} \mathrm{~A}$. Szöke and E. Courtens, Phys. Rev. Lett. 34, 1053 (1975). The concept of adiabatic excitation introduced in this paper is closely related to coherent excitation.

${ }^{22}$ David Bohm, Quantum Theory (Prentice-Hall, Englewood Cliffs, 1951), p. 450.

${ }^{23}$ Albert Messiah, Quantum Mechanics (North-Holland, Amsterdam, 1962), Vol. II, p. 740.

${ }^{24}$ G. Lochak, C. R. Acad. Sci. B 272, 1281 (1971); 274, 1391 (1972); 275, 49 (1972); 275, 903 (1972); 276, 103
(1973); 276, 809 (1973); 279, 547 (1974); A. Alaoui and G. Lochak, ibid. 280, 589 (1975).

${ }^{25} \mathrm{C}$. H. Townes and A.L. Schawlow, Microwave Spectroscopy (McGraw-Hill, New York, 1955), p. 273.

${ }^{26}$ W. Happer, Phys. Rev. 136, A35 (1964).

${ }^{27}$ D. Guichon, G. Lochak, G. Theobald, and J. G. Theobald, C. R. Acad. Sci. B 274, 935 (1972).

${ }^{28} \mathrm{~A}$. Szöke and E. Courtens (unpublished).

${ }^{29}$ R. P. Feynman, F.L. Vernon, Jr., and R. W. Hellwarth, J. Appl. Phys. 28, 49 (1957).

${ }^{30} \mathrm{Ch}$ 'en Shang-Yi, Phys. Rev. 58, 884 (1940).

${ }^{31} \mathrm{~W}$. Heitler, The Quantum Theory of Radiation, 3rd ed. (Oxford U. P., London, 1954), pp. 196-204.

${ }^{32}$ B. R. Mollow, Phys. Rev. 188, 1969 (1969); Phys. Rev. A 2, 76 (1970).

${ }^{33}$ A. P. Kazantsev, Zh. Eksp. Teor. Fiz. 66, 1229 (1974) [Sov. Phys.-JETP 39, 601 (1974)]

${ }^{34}$ R. H. Lehmberg and J. Reintjes, Phys. Rev. A 12 , 2574 (1975). These authors show that when $T_{2}=2 \tau_{s}$ and $T_{1}=\tau_{s}$, then $N_{n}=0$. However, for our case here, $T_{2}$ $\ll T_{1}$ due to radiative trapping, and consequently the paper by Lehmberg and Reintjes appears to predict substantial $N_{n}(t)$ in the absence of collisions, although this situation was not explicitly considered.

${ }^{35}$ J. L. Carlsten and A. Szöke, Phys. Rev. Lett. 36, 667 (1976)

${ }^{36} \mathrm{~J}$. A. Armstrong, N. Bloembergen, J. Ducuing, and P. S. Pershan, Phys. Rev. 127, 1918 (1962).

${ }^{37}$ A. M. Bonch-Bruevich and V. A. Khodovoi, Usp. Fiz. Nauk 93, 71 (1967) [Sov. Phys.-Usp. 10, 637 (1968)].

${ }^{38} \mathrm{C}$. Cohen-Tannoudji, in Cargese Lectures in Physics, edited by M. Levy (Gordon and Breach, New York, 1968), Vol. 2, p. 347.

${ }^{39} \mathrm{~S}$. Haroche, Ann. Phys. (Paris) 6, 189 (1971).

${ }^{40} \mathrm{~A}$. Flusberg and S. R. Hartmann, following paper, Phys. Rev. A 14, 813 (1976).

${ }^{41}$ W. A. Anderson, Phys. Rev. 102, 151 (1956).

${ }^{42} \mathrm{~J}$. A. Armstrong and D. Grischkowsky, U. S. Patent No. 3,864,020 (1975).

${ }^{43}$ P. F. Liao and G. C. Bjorklund, Phys. Rev. Lett. 36 , 584 (1976).

${ }^{44}$ W. K. Bischel, P. J. Kelly, and C. K. Rhodes, Phys. Rev. A 13, 1817 (1976). See Eq. (21).

${ }^{45}$ Because of the Zeeman splitting and the use of circularly polarized light the effective number density is one-half of the total number density.

${ }^{46} \mathrm{~W}$. Happer (private communication).

${ }^{47}$ M. D. Crisp, Phys. Rev. A 1, 1604 (1970).

${ }^{48}$ I. D. Abella, Phys. Rev. Lett. 9, 453 (1962).

${ }^{49}$ D. Pritchard, J. Apt, and T. W. Ducas, Phys. Rev. Lett. 32,641 (1974).

${ }^{50}$ F. Biraben, B. Cagnac, and G. Grynberg, Phys. Rev. Lett. 32,643 (1974).

${ }^{51}$ M. D. Levenson and N. Bloembergen, Phys. Rev. Lett. 32,645 (1974).

${ }^{52}$ T. W. Hänsch, K. C. Harvey, G. Meisel, and A. L. Schawlow, Opt. Commun. 11, 50 (1974).

${ }^{53}$ J. E. Bjorkholm and P. F. Liao, Phys. Rev. Lett. 3.3 , 128 (1974).

${ }^{54}$ P. F. Liao and J. E. Bjorkholm, Phys. Rev. Lett. $\underline{34}$, 1 (1975). 

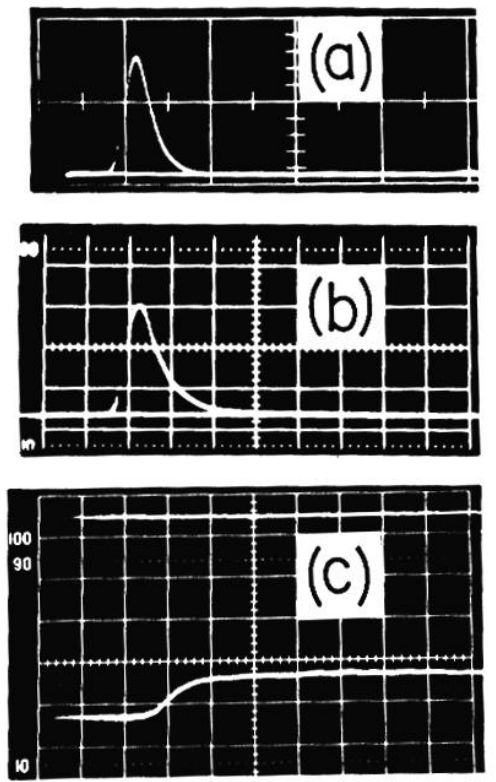

FIG. 4. Experimental measurement of the incoherent excitation $N_{n}(t)$. (a) Input $\overrightarrow{\mathrm{E}}_{2}$ pulse to the $\mathrm{Rb}$ cell with the peak intensity of $200 \mathrm{~W} / \mathrm{cm}^{2}$ and the frequency offset $\Delta \omega=0.8 \mathrm{~cm}^{-1}$. The pulse was measured with an ITT biplanar photodiode and Tektronix 519 oscilloscope. Sweep speed is 10 nsec per large division. (b) Corresponding output pulse measured with an ITT biplanar photodiode and a Tektronix 7904 oscilloscope. Sweep speed is 5 nsec per large division. (c) Transient absorption of the weak $\vec{E}_{1}$ probing beam tuned to the singlephoton resonance $\omega_{t}=\Omega_{1 n}$. Top trace is the zero-intensity baseline. Bottom trace is the cw signal, which shows transient absorption due only to $N_{n}(t)$. The signal was measured with a Spectra-Physics 403 photodiode and a Tektronix 7904 oscilloscope. Sweep speed is $5 \mathrm{nsec}$ per large division. 



FIG. 5. Experimental measurement of the coherent excitation $N_{n}^{*}(t)$. (a) Input pulse to the $\mathrm{Rb}$ cell with the peak intensity of $500 \mathrm{~W} / \mathrm{cm}^{2}$ and the frequency offset $\Delta \omega$ $=0.6 \mathrm{~cm}^{-1}$. The pulse was measured with an ITT biplanar photodiode and a Tektronix 519 oscilloscope. Sweep speed is 10 nsec per large division. (b) Transient absorption of the weak $\overrightarrow{\mathrm{E}}_{1}$ probing beam tuned to the two-photon resonance $\omega_{1}+\omega_{2}=\Omega_{12}$. Top trace is the zero-intensity baseline. Bottom trace is the cw signal which shows transient absorption due only to $N_{n}^{*}(t)$. The signal was measured with a Spectra-Physics 403 photodiode and a Tektronix 7904 oscilloscope. Sweep speed is 5 nsec per large division. 

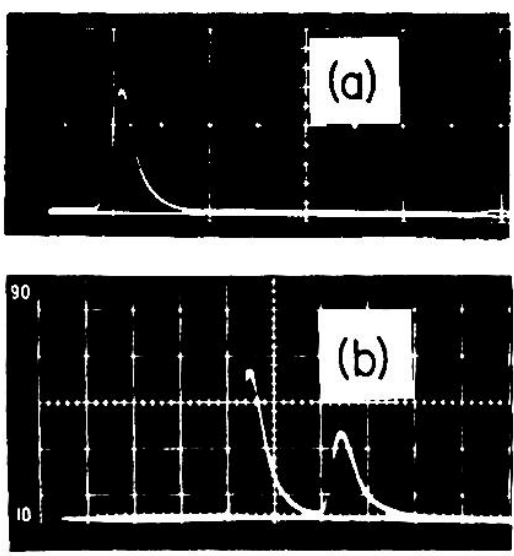

FIG. 6. (a) Input linearly polarized $\overrightarrow{\mathrm{E}}_{2}$ pulse to the $100-\mathrm{cm} \mathrm{Rb}$ cell with the peak intensity of $6 \mathrm{~W} / \mathrm{cm}^{2}$. The pulse was measured with an ITT biplanar photodiode and a Tektronix 519 oscilloscope. Sweep speed is $10 \mathrm{nsec}$ per large division. (b) Resulting two circularly polarized $\sigma^{+}$and $\sigma^{-}$output pulses from the cell. The pulses were monitored with an ITT biplanar photodiode and a Tektronix 7904 oscilloscope. The sweep speed is 5 nsec per large division, giving the measured pulse separation of $9.5 \mathrm{nsec}$, corresponding to the group velocities of $v_{g}^{-}=\frac{1}{4} c$ and $v_{g}^{+}=c / 1.2$ for the $\sigma^{-}$and $\sigma^{+}$pulses, respectively. The $\mathrm{Rb}$ cell temperature was $140^{\circ} \mathrm{C}$ and the cell was in a magnetic field of $9 \mathrm{kG}$. The frequency $\omega_{2}$ of the laser light was $0.4 \mathrm{~cm}^{-1}$ below the center frequency of the $\sigma^{-}$hyperfine components of the Zeemansplit $\Omega_{n 2}$ resonance line and $1.5 \mathrm{~cm}^{-1}$ below the center of the $\sigma^{+}$components. 